\title{
Ulinastatin protects against sepsis-induced myocardial injury by inhibiting NLRP3 inflammasome activation
}

\author{
JUANJUAN QIU $^{1 *}$, XIAOGUANG XIAO ${ }^{2 *}, \mathrm{XUE} \mathrm{GAO}^{3}$ and YONGLI ZHANG ${ }^{4}$ \\ ${ }^{1}$ Centralab; ${ }^{2}$ Clinical Laboratory; Departments of ${ }^{3}$ Pathology and ${ }^{4}$ Critical Care Medicine, \\ The First Affiliated Hospital of Dalian Medical University, Dalian, Liaoning 116011, P.R. China
}

Received March 27, 2021; Accepted July 22, 2021

DOI: $10.3892 / \mathrm{mmr} .2021 .12369$

\begin{abstract}
Myocardial injury is the primary manifestation of multiple organ dysfunction during sepsis, however, the mechanisms underlying sepsis-induced myocardial injury remain unclear. Similarly, no effective therapeutics have yet been developed for myocardial injury. In the present study, the role of the NOD-like receptor 3 (NLRP3) inflammasome on cardiac function were characterized and the effects of different ulinastatin (UTI) doses in protecting a septic rat model from myocardial injury were elucidated. To evaluate UTI efficacy on cardiac function, its effects on anti-inflammatory mediators were analyzed and its cardioprotective effects were investigated. It was demonstrated that circulatory levels of tumor necrosis factor- $\alpha$ and interleukin-1 $\beta$ were elevated during sepsis. It was also observed that NLRP3 and caspase-1 expression enhanced post-cecal ligation and puncture (CLP), and that high UTI levels protected against myocardial injury induced by sepsis. To the best of our knowledge, this is the first study to demonstrate that the mechanisms underpinning UTI-mediated myocardial protection were due to the downregulation of the NLRP3/caspase-1/IL-1 $\beta$ signaling pathway. Based on these findings, it is proposed that UTI exerts beneficial effects during sepsis-induced myocardial injury.
\end{abstract}

Correspondence to: Dr Yongli Zhang, Department of Critical Care Medicine, The First Affiliated Hospital of Dalian Medical University, 222 Zhongshan Road, Dalian, Liaoning 116011, P.R. China

E-mail: zylicu@163.com

Dr Xue Gao, Department of Pathology, The First Affiliated Hospital of Dalian Medical University, 222 Zhongshan Road, Dalian, Liaoning 116011, P.R. China

E-mail: xueer_128@163.com

*Contributed equally

Key words: sepsis, cecal ligation and puncture, myocardial injury, ulinastatin, NOD-like receptor 3 inflammasome

\section{Introduction}

Sepsis is a severe life-threatening organ dysfunction condition caused by a dysregulated host response to infection (1). Sepsis, with accompanying systemic inflammatory response syndrome, affects multiple systems and organs and culminates in multiple organ dysfunction syndrome (2). Myocardial injury is a major and serious complication of sepsis; sepsis-induced myocardial injury has a high incidence rate and is observed in $40-50 \%$ of patients with sepsis (3). Moreover, studies have reported that sepsis-induced cardiac dysfunction may be as high as $70 \%$ (4) and is a major predictor of mortality from sepsis (5). However, current treatments for myocardial injury following sepsis are not ideal, as mechanisms underpinning sepsis-induced myocardial injury are not fully understood.

Inflammasomes are multi-protein cellular complexes that activate inflammatory cascades (6). Currently, the NOD-like receptor 3 (NLRP3) inflammasome is the most characterized and widely described inflammasome complex and comprises NLRP3, the adaptor protein, ASC (also known as PYCARD) and caspase-1 (7). Increasingly, evidence has indicated that activated NLRP3 triggers caspase-1, which then triggers mature interleukin-1 $\beta$ (IL-1 $\beta$ ) production and secretion $(8,9)$. IL- $1 \beta$ is a vital cytokine that mediates the secretion of large quantities of other inflammatory mediators and proinflammatory cytokines that damage multiple organs $(10,11)$. Thus, the NLRP3/caspase- $1 / \mathrm{IL}-1 \beta$ axis exerts significant proinflammatory effects. At the molecular level, the NLRP3 inflammasome is activated during sepsis, with high expression of the NLRP3/caspase-1/IL-1 $\beta$ signaling pathway (12). Although some studies have reported NLRP3 is essential for bacterial clearance with protective roles in infectious diseases (13), it is currently unclear whether NLRP3 inflammasome activation exerts protective or detrimental roles during sepsis-induced myocardial injury. Targeting anti-inflammatory mediators is a promising therapeutic strategy for preventing and treating sepsis-induced organ dysfunction, however, little success has been achieved as the biological process is highly complex. Several studies have reported that NLRP3 inhibitors specifically target in vivo NLRP3 activation (14-16). Thus, it is conceivable to develop novel drugs to treat or prevent septic organ injury that not only target inflammatory mediators, but 
also NLRP3, thereby eliminating the main inflammatory response activator (17).

Ulinastatin, also called urinary trypsin inhibitor (UTI), is a broad-spectrum protease inhibitor derived from human urine that inhibits multiple endogenous proteases (18). It exhibits a protective effect via anti-inflammatory and anti-oxidative stress responses (19). Previous studies have shown that UTI exerts organ-protective effects during sepsis $(20,21)$. However, whether UTI suppresses NLRP3 inflammasome activation, downregulates the expression of related mediators, and elicits protective effects toward myocardial injury induced by sepsis remains largely unknown.

In the present study, animal studies were conducted to investigate NLRP3 inflammasome mechanisms in sepsis-induced myocardial injury and evaluate the effects of UTI on injured myocardium in septic rats.

\section{Materials and methods}

Animals. A total of 75 male Wistar rats weighing 180-200 g (age, 7-9 weeks old) were purchased from the Laboratory Animal Center of Dalian Medical University (Dalian, China) and housed under controlled temperatures $\left(20-25^{\circ} \mathrm{C}\right)$ and humidity (2-30\%) under a $12 \mathrm{~h}$ light/dark cycle. Rats were allowed access to food and water ad libitum. This research was approved by the Ethics Committee of Dalian Medical University (approval no. L20160097) and was performed in accordance with the National Institute of Health guidelines for ethical animal research (22).

Study design and UTI use. Rats $(\mathrm{n}=75)$ were randomly divided into five groups, with 15 rats per group: i) Control; ii) sham-operation; iii) cecal ligation and puncture (CLP); iv) CLP plus low-dose UTI $(5,000 \mathrm{U} / \mathrm{kg})$; and v) CLP plus high-dose UTI $(20,000 \mathrm{U} / \mathrm{kg})$. UTI was provided by Guangdong Techpool Bio-Pharma Co. Ltd.

CLP. CLP procedures were performed according to a previous study by Rittirsch et al (23) to generate a sepsis model. Animals were anesthetized with an intraperitoneal injection of $1 \%$ pentobarbital sodium $(50 \mathrm{mg} / \mathrm{kg}$; Shanghai Ling Feng Chemical Reagent Co., Ltd.) before a midline laparotomy. Abdominal fur was removed, the area disinfected and a sterile towel was spread over the area. A 1-cm midline incision along the abdomen midline was performed, the cecum was located and carefully isolated to avoid vascular injury. Approximately $1 / 3$ of the entire cecum was ligated with a No. 4 suture on the distal side of the ileocecal valve. Then, the distal cecum was punctured twice with a sterile 18-gauge needle and a small amount of intestinal contents was squeezed out (24). After the cecum was placed back into the abdominal cavity, the abdominal wall incision was sutured layer by layer. Rats were resuscitated with a saline solution administered by subcutaneous injection ( $3 \mathrm{ml} / 100 \mathrm{~g}$ bodyweight). Sham-operation animals were treated the same way, but no cecal ligation or puncture was performed. Control group animals were not surgically processed, but were resuscitated with subcutaneous saline. Animal spontaneous activity, reaction to exogenous stimuli and posture were observed. Clinical severity scores were calculated every $6 \mathrm{~h}$ to evaluate the clinical state and sepsis severity was determined by two experienced observers blinded to treatments. All assessment scores were collected for analysis (25).

Survival observations. Animals were observed for $48 \mathrm{~h}$ after surgery, with survival status recorded and survival rate comparisons made between different groups. Rats were sacrificed if they demonstrated any of the following characteristics: Loss of spontaneous respiratory movement and heartbeat for a period of $2 \mathrm{~min}$, a moribund state and/or hypothermia (rectal temperature $<32^{\circ} \mathrm{C}$ ).

Administration of UTI and specimen retention. After surgery, at $0,6,12,18$ and $24 \mathrm{~h}$, rats assigned to low- and high-dose UTI groups were treated with 5,000 and $20,000 \mathrm{U} / \mathrm{kg}$ (intraperitoneal injection) UTI, respectively. Control, sham-operation and CLP groups were injected with the same volume of normal saline. At $48 \mathrm{~h}$ post-surgery and before animals were sacrificed, blood from the angular vein was collected into sodium citrate tubes. At the study end, surviving rats in each group were humanely euthanized by cervical dislocation. Tissues from the left ventricle were harvested, with samples placed in buffered formalin, liquid nitrogen and frozen at $-80^{\circ} \mathrm{C}$. A study flowchart is shown in Fig. 1.

Inflammatory markers and myocardial injury measurement. Blood samples from time points were immediately centrifuged at $3,000 \mathrm{x} \mathrm{g}$ for $15 \mathrm{~min}$ at room temperature. The serum was removed and stored at $-80^{\circ} \mathrm{C}$ until enzyme-linked immunosorbent assays (ELISA) were performed. Then, tumor necrosis factor- $\alpha$ (TNF- $\alpha)$, IL-1 $\beta$, cardiac troponin I (cTnI) and B-type natriuretic peptide (BNP) serum levels were measured by commercial ELISA kits as per the manufacturer's instructions. cTnI serum levels were expressed as $\mu \mathrm{g} / \mathrm{ml}$, whereas BNP, TNF- $\alpha$ and IL-1 $\beta$ were expressed as $\mathrm{pg} / \mathrm{ml}$. ELISA kits for TNF- $\alpha$ (cat. no. ab236712) and IL-1 $\beta$ (cat. no. ab255730) quantification were obtained from Abcam, cTnI ELISA kits (cat. no. BPE30309) and BNP ELISA kits (cat. no. BPE30445) were purchased from Shanghai Lengton Bioscience Co., Ltd.

Myocardial histology. For hematoxylin and eosin (H\&E) staining, left ventricle apical tissue was collected and immediately washed twice in phosphate-buffered saline (PBS, pH 7.4) to remove blood. The tissue was fixed in $10 \%$ neutral formalin for $72 \mathrm{~h}$ at $25^{\circ} \mathrm{C}$. Then, samples were successively dehydrated (successive immersion in 75, 85, 90 and 95\% alcohol, anhydrous acetic acid, anhydrous ethanol and anhydrous ethanol for $30 \mathrm{~min}$ each) and paraffin embedded. Tissue sections $(4 \mu \mathrm{m})$ were then fixed in ethanol as follows: Immersed in 70, 80 and $90 \%$ ethanol for 4-5 sec, and immersed in anhydrous ethanol for $5 \mathrm{~min}$ at room temperature. The sections were stained with hematoxylin for $5 \mathrm{~min}$ and eosin solution for $3 \mathrm{~min}$ at room temperature, followed by dehydration with graded alcohol and clearing in xylene. Stained sections were then analyzed and imaged using light microscopy (magnification, x400; Olympus Corporation).

Transmission electron microscopy. Left ventricle apical tissues were fixed in cold $2.5 \%$ glutaraldehyde for $12 \mathrm{~h}$, post-fixed in $1 \%$ osmium tetroxide for $1 \mathrm{~h}$ at room temperature, dehydrated 
Table I. Primer sequences used for reverse transcription-quantitative PCR.

\begin{tabular}{lll}
\hline Genes & \multicolumn{1}{c}{ Forward primers $\left(5^{\prime} \rightarrow 3^{\prime}\right)$} & Reverse primers $\left(5^{\prime} \rightarrow 3^{\prime}\right)$ \\
\hline NLRP3 & CAGCGATCAACAGGCGAGAC & AGAGATATCCCAGCAAACCTATCCA \\
Caspase-1 & ACTCGTACACGTCTTGCCCTCA & CTGGGCAGGCAGCAAATTC \\
GADPH & GCACCGTCAAGGCTGAGAAC & TGGTGAAGACGCCAGTGGA \\
\hline
\end{tabular}

NLRP3, NOD-like receptor 3.

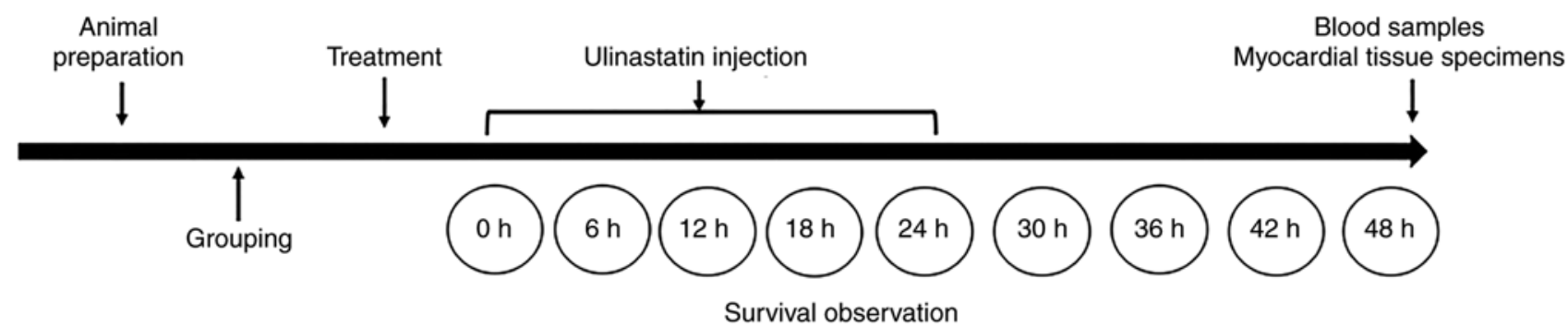

Figure 1. Flowchart showing the experimental procedures of the present study.

at room temperature and embedded in Epon for $12 \mathrm{~h}$ at $45^{\circ} \mathrm{C}$. Ultrathin sections $(60-80 \mathrm{~nm})$ were randomly cut and stained with lead citrate for $10 \mathrm{~min}$ at room temperature and uranyl acetate for $30 \mathrm{~min}$ at room temperature. Transmission electron microscopy (JEM-2000EX; JEOL, Ltd.) was used to examine myocardium structures between $\mathrm{x} 10,000$ and $\mathrm{x} 30,000$ magnification. Five electron micrographs were randomly and sequentially obtained from one section per animal.

Reverse transcription-quantitative (RT-q)PCR. NLRP3 and caspase-1 mRNA expression levels in the left ventricular myocardium were determined via RT-qPCR. Total mRNA was extracted from tissue using RNAiso Plus (Takara Biotechnology Co., Ltd.) and reverse transcribed to cDNA using a PrimeScript ${ }^{\mathrm{TM}}$ RT reagent kit (Takara Biotechnology Co., Ltd.) with gDNA Eraser according to the manufacturer's instructions. RT-qPCR was conducted using SYBR ${ }^{\circledR}$ Premix Ex $\mathrm{Taq}^{\mathrm{TM}}$ II kit (Takara Biotechnology Co., Ltd.) following the manufacturer's protocols. The RT-qPCR procedure was conducted as follows: One cycle of $95^{\circ} \mathrm{C}$ for $30 \mathrm{sec} ; 40$ cycles of $95^{\circ} \mathrm{C}$ for $5 \mathrm{sec}$ and $60^{\circ} \mathrm{C}$ for $30 \mathrm{sec}$. All samples were analyzed in triplicate. Relative expression was calculated using the $2^{-\triangle \Delta \mathrm{Cq}}$ method with GAPDH as an endogenous reference (26). All RT-qPCR primer sequences are shown in Table I.

Western blotting. NLRP3 and caspase-1 protein expression levels in the left ventricular myocardium were determined by western blotting. Tissues were homogenized in lysis reagent (Sigma-Aldrich; Merck KGaA) supplemented with phenylmethylsulfonyl fluoride (Beyotime Institute of Biotechnology), and protein concentrations were determined using a bicinchoninic acid assay kit (Sangon Biotech Co., Ltd.). Total protein was separated via $8 / 10 \%$ polyacrylamide gels, and subsequently transferred to polyvinylidene difluoride membranes. Primary antibodies against $\beta$-actin (1:1,000; cat. no. KC5A08; Kangchen BioTech Co., Ltd.), anti-caspase-1 (1:1,000; cat. no. SC-56036; Santa Cruz Biotechnology, Inc.), and anti-NLRP3 (1:1,000; cat. no. 19771-1-AP; ProteinTech Group, Inc.). Blots were blocked in $5 \%$ milk for $2 \mathrm{~h}$ at $37^{\circ} \mathrm{C}$, incubated overnight at $4^{\circ} \mathrm{C}$ with primary antibodies and washed in PBS with $0.05 \%$ Tween-20 for $20 \mathrm{~min}$. Followed by incubation with a goat anti-rabbit secondary antibody $(1: 1,000$; ProteinTech Group, Inc.) at room temperature for $2 \mathrm{~h}$. Protein bands were visualized using enhanced chemiluminescence (Applygen Technologies, Inc.) and semi-quantified using ImageJ software (version 1.8.0; National Institutes of Health).

Statistical analysis. All experiments were repeated three times. All statistical analyses were performed using SPSS 20.0 (IBM Corp.). Values are expressed as the mean \pm standard deviation. Data were analyzed using a two-tailed unpaired Student's t-test to compare two groups. Comparisons among multiple groups were conducted using one-way ANOVA with post hoc Bonferroni test. Survival rates over time were conducted using Kaplan-Meier and log-rank analyses. $\mathrm{P}<0.05$ was considered to indicate a statistically significant difference.

\section{Results}

UTI enhances survival in a rat sepsis model. Kaplan-Meier survival curves and clinical severity score curves were used to determine the protective effects of UTI on survival rates and sepsis-related symptom alleviation. Kaplan-Meier survival curves at $48 \mathrm{~h}$ are shown for control, sham, CLP, low- and high-dose UTI animals (Fig. 2A). Control and sham animals appeared normal with all surviving; blue and orange curves for both corresponded to a $100 \%$ survival rate. However, the CLP group showed a steep decline in survival after surgery; most animals died from septic shock within 10-40 h. The survival rate of CLP, low- and high-dose UTI rats were 53.3, 80.0 and 86.7\%, respectively. The UTI groups exhibited significantly improved survival rates compared with the CLP group ( $<<0.01$; Fig. $2 \mathrm{~A})$. 

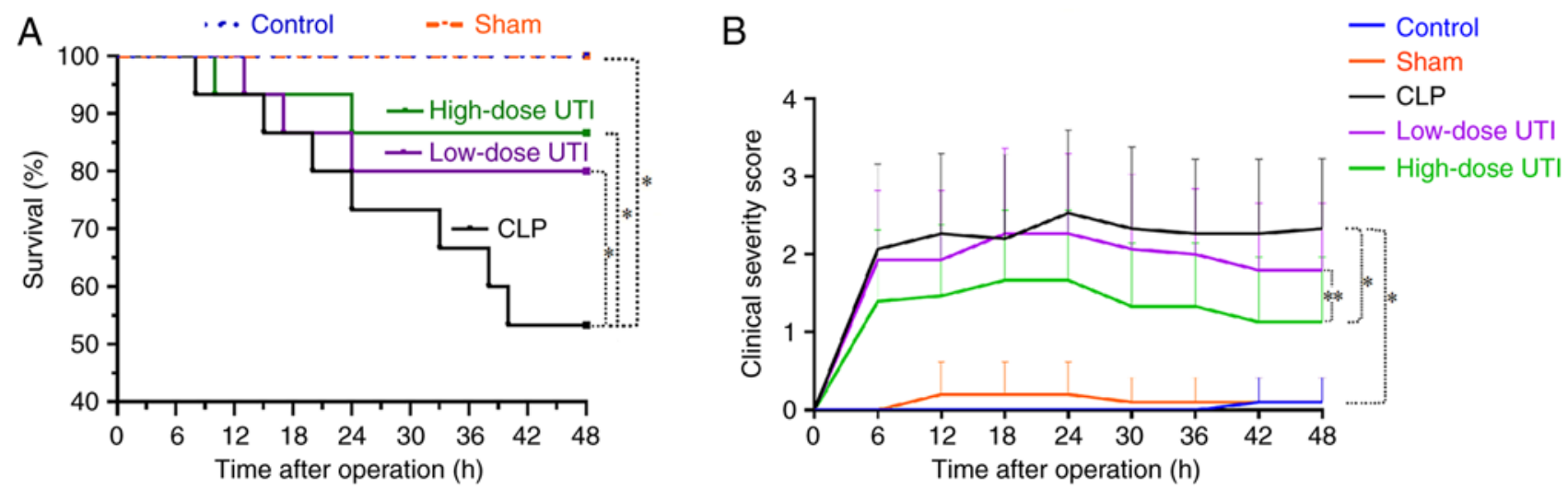

Figure 2. UTI improves the survival rates and health status of septic rats. UTI was administered to rats after CLP. No rats died in the control and sham groups, seven rats died in the CLP group, three rats died in the low-dose UTI group and two rats died in the high-dose UTI group. (A) Rat mortality was monitored every $6 \mathrm{~h}$ and the percent survival rate was expressed as a Kaplan-Meier survival curve ( $\mathrm{n}=15$ per group). (B) Clinical scores evaluating the health status of surviving rats. Data are expressed as the mean \pm standard deviation. ${ }^{*} \mathrm{P}<0.01,{ }^{* *} \mathrm{P}<0.05$. UTI, ulinastatin; CLP, cecal ligation and puncture.

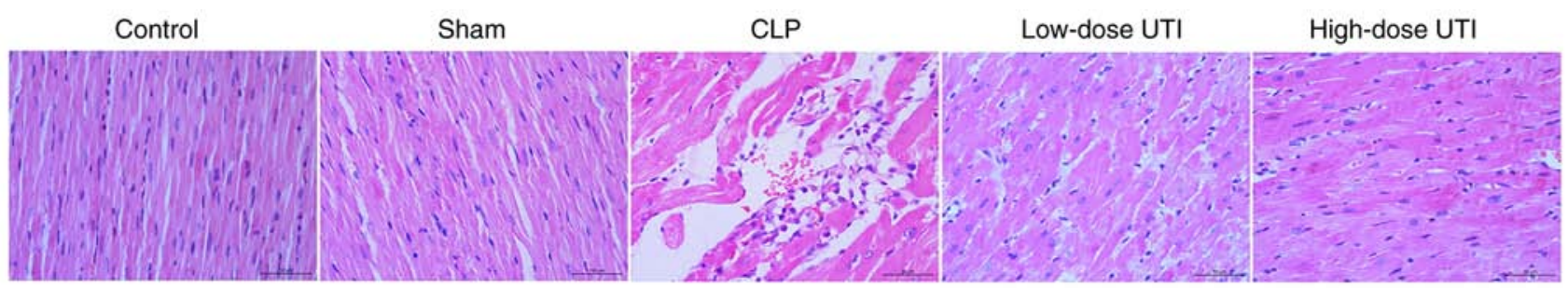

Figure 3. Hematoxylin and eosin staining of myocardium tissue. Control group showed normal histology. The sham-operation group displayed almost normal tissue structure and shape, the myocardial fibers were arranged in an orderly pattern and there was an absence of cardiomyocyte necrosis and inflammatory cell infiltration. The CLP group showed disrupted myocardial fibers, severe swelling and necrosis of myocardial cells, and heavy neutrophil and macrophage infiltration. The low-dose UTI group displayed edema and leukocyte infiltration, while the high-dose UTI group showed mild edema and leukocyte infiltration. Magnification, x400. UTI, ulinastatin; CLP, cecal ligation and puncture.

Clinical severity score curves revealed a delayed response to CLP operation stimuli and peaked at $24 \mathrm{~h}$. Scores in the CLP group were significantly higher than both UTI treatment groups; clinical severity scores in the low-dose UTI group were slightly higher than those in the high-dose UTI group $(\mathrm{P}<0.05$; Fig. 2B). These findings suggested that UTI potentially alleviated sepsis-related symptoms and reduced mortality rate in a septic rat model.

UTI alleviates myocardial tissue damage. Using light microscopy, H\&E myocardial stained sections were used to investigate myocardial tissue damage and inflammatory cell infiltration, whereas ultra-structural and mitochondrial changes were examined using transmission electron microscopy.

In the control group, myocardial cell morphology was intact with a regulardistribution and normal mitochondrialmorphology. Sham-group observations were similar. By contrast, the CLP group displayed a scattered coagulated myocardial cell necrosis, massive neutrophil infiltration between cardiomyocytes, and blurred nuclear and organizational structures. Pathological changes in the low- and high-dose UTI groups appeared less severe than CLP animals. By contrast, cell morphology in the high-dose UTI group had significantly improved, and only slightly improved in the low-dose UTI group (Fig. 3).

Myocardial morphological structures and mitochondrial crista were ordered and clear in control and sham groups.
However, in the CLP group, mitochondria were significantly swollen, myocardial fibers were disordered, broken and dissolved. By contrast, in UTI groups, especially the high-dose group, heart morphological structures were improved and mitochondrial crista was clearer (Fig. 4). These structural changes indicated that UTI potentially exerted cardioprotective effects by suppressing cardiac inflammation in CLP-treated rats.

UTI suppresses inflammatory mediator release and attenuates myocardial injury. In response to $48 \mathrm{~h}$ post-surgery sepsis mediated inflammatory marker level increases in serum, significant increases in TNF- $\alpha$ and IL-1 $\beta$ serum levels were observed in the CLP group, moderate increases in the low-dose UTI group and mild increases in the high-dose UTI group compared with the control and sham groups $(\mathrm{P}<0.01)$. No statistically significant differences were found between control and sham groups $(\mathrm{P}>0.05)$. Compared with the CLP group, TNF- $\alpha$ and IL-1 $\beta$ serum levels were significantly decreased in the low- and high-dose UTI groups $(\mathrm{P}<0.01)$, and significantly decreased in the high-dose group compared with the low-dose group ( $\mathrm{P}<0.05$; Fig. 5A and $\mathrm{B})$. Based on these comparisons, it was concluded UTI treatments inhibited inflammatory responses during sepsis, particularly at the higher dose.

cTnI and BNP serum levels are indicators of myocardial injury, therefore, to test the effects of UTI on sepsis-induced myocardial injury, the serum levels of these myocardial injury 


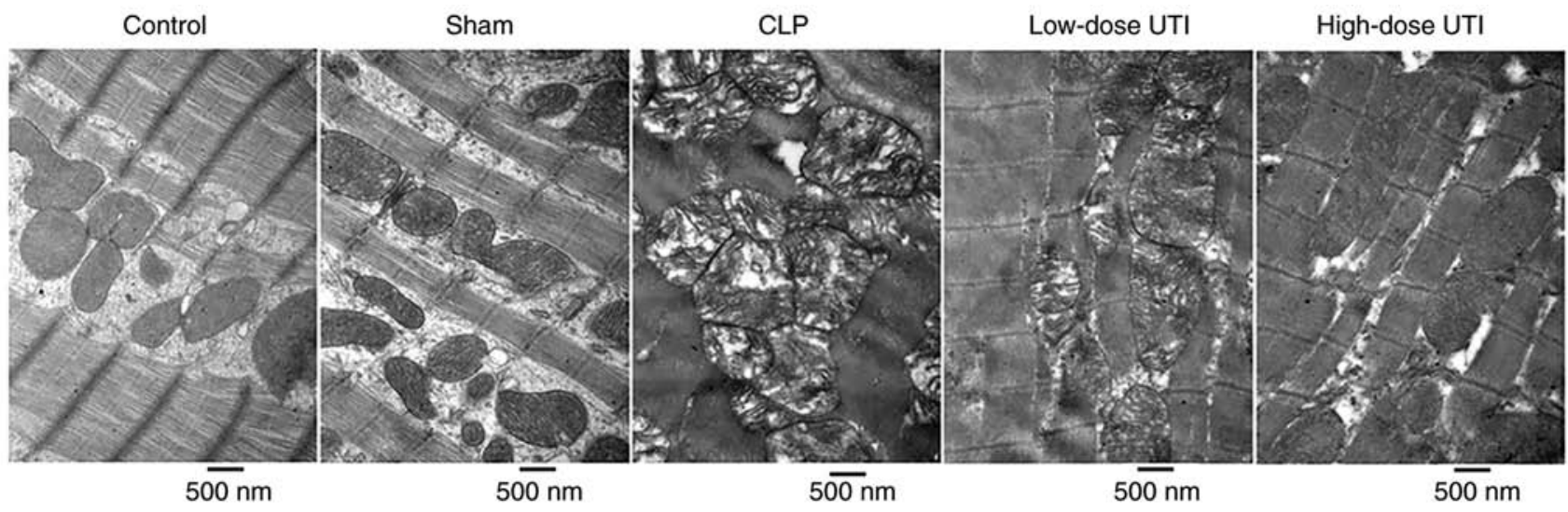

Figure 4. Myocardial tissue observations under a transmission electron microscope. Control and sham groups showed regularly arranged myocardial tissue without swollen mitochondria. Mitochondrial membrane structures were clear. In the CLP group, myocardial fibers were irregularly arranged or disrupted, with myocardial cell swelling. Most mitochondria were severely swollen, broken and dissolved. Vacuolization was also visible in mitochondria. In the low-dose UTI group, the myocardial structures were complete and mitochondrial membrane structures had partially disappeared. Only light vacuolization was observed in some mitochondria. In the high-dose UTI group, myocardial structures had improved, mitochondria were slightly swollen and membrane structures were clear. Magnification, x30,000. UTI, ulinastatin; CLP, cecal ligation and puncture.
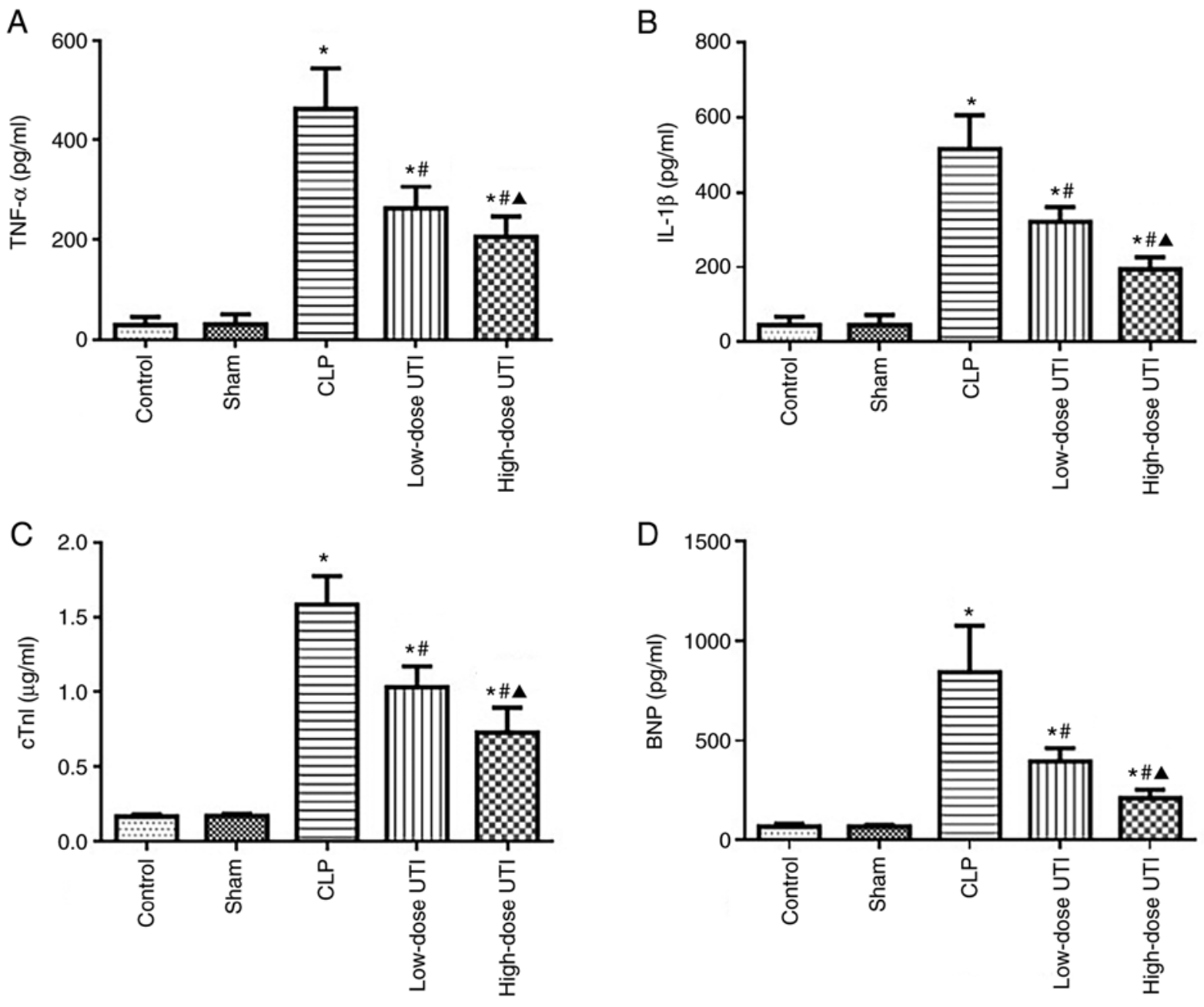

Figure 5. Expression of inflammatory biomarkers and myocardial injury markers. (A) TNF- $\alpha$ serum levels. (B) IL-1 $\beta$ serum levels. (C) cTnI serum levels. (D) BNP serum levels. $\mathrm{n}=15$ in each group. Studies were repeated three times with highly similar results. ${ }^{*} \mathrm{P}<0.01$ vs. control and sham groups; ${ }^{*} \mathrm{P}<0.01 \mathrm{vs}$. CLP group; ${ }^{\wedge} \mathrm{P}<0.05$ vs. low-dose UTI group. TNF- $\alpha$, tumor necrosis factor- $\alpha$; IL-, interleukin; UTI, ulinastatin; CLP, cecal ligation and puncture; cTnI, cardiac troponin I; BNP, B-type natriuretic peptide.

markers were examined at $48 \mathrm{~h}$ post-surgery. Significant increases in cTnI and BNP serum levels were observed in the
CLP group, moderate increases in the low-dose UTI group and mild increases in the high-dose UTI group compared 

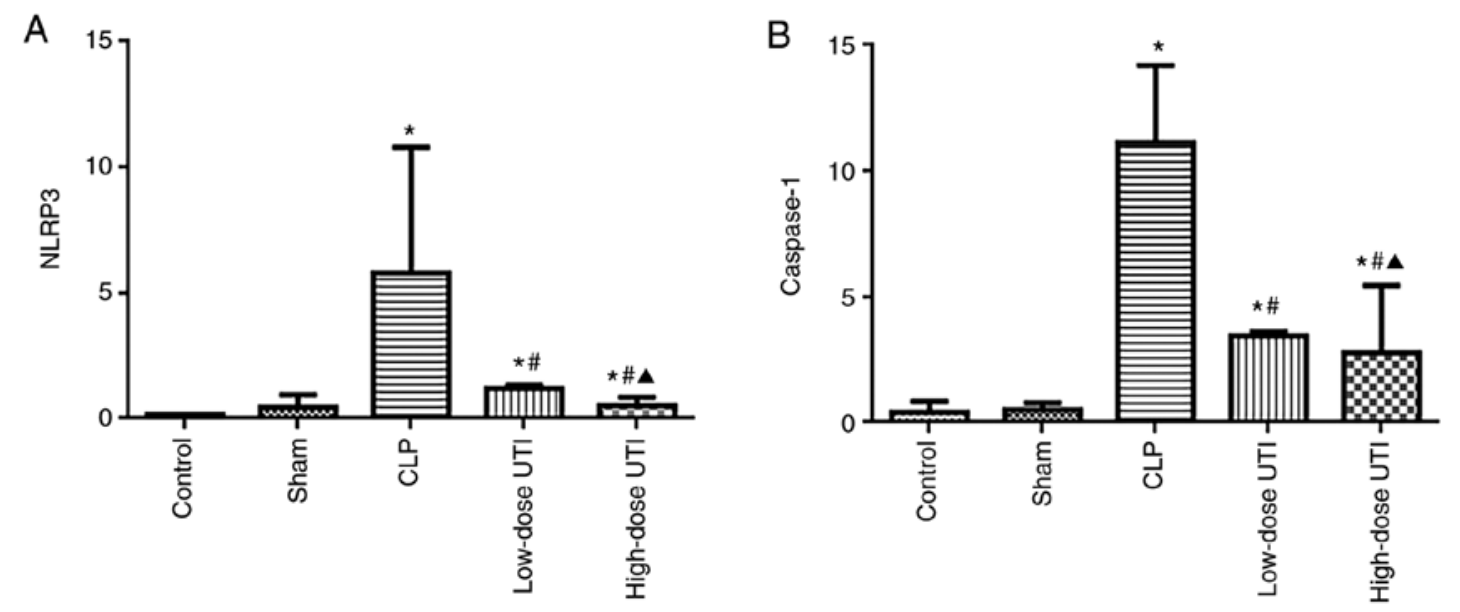

Figure 6. NLRP3 and caspase-1 mRNA expression in the left ventricular myocardium. (A) NLRP3 and (B) caspase-1 mRNA expression levels. Levels were normalized to GAPDH. $\mathrm{n}=15$ in each group. Studies were repeated three times with highly similar results. ${ }^{*} \mathrm{P}<0.01$ vs. control and sham groups; ${ }^{\prime \prime} \mathrm{P}<0.01$ vs. CLP group; ${ }^{\wedge} \mathrm{P}<0.05$ vs. low-dose UTI group. UTI, ulinastatin; CLP, cecal ligation and puncture; NLRP3, NOD-like receptor 3.

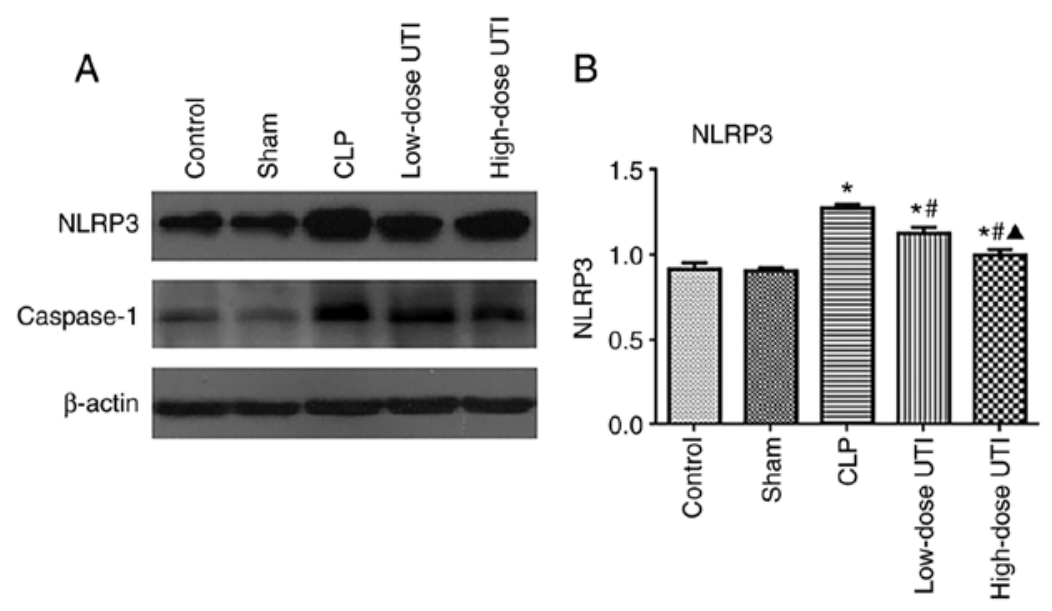

C

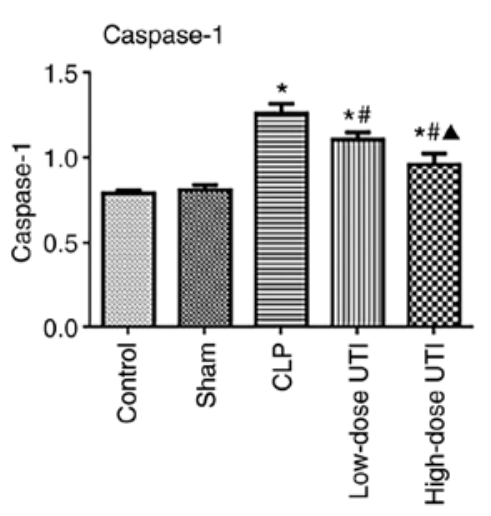

Figure 7. NLRP3 and caspase-1 protein expression in the left ventricular myocardium. (A) NLRP3 and caspase-1 protein expression levels normalized to $\beta$-actin. Semi-quantitative analysis of (B) NLRP3 and (C) caspase-1 protein expression levels. $n=15$ in each group. Studies were repeated three times with highly similar results. "P $<0.01$ vs. control and sham groups; ${ }^{\#} \mathrm{P}<0.01$ vs. CLP group; ${ }^{\mathbf{}} \mathrm{P}<0.05$ vs. low-dose UTI group. UTI, ulinastatin; CLP, cecal ligation and puncture; NLRP3, NOD-like receptor 3.

with the control and sham groups $(\mathrm{P}<0.01)$. No statistically significant differences were observed between control and sham groups $(\mathrm{P}>0.05)$. Compared with the CLP group, cTnI and BNP serum levels were significantly decreased in the lowand high-dose UTI groups $(\mathrm{P}<0.01)$, while in the high-dose group levels were significantly decreased compared with the low-dose group ( $\mathrm{P}<0.05$; Fig. 5C and D). Taken together, these observations demonstrated that UTI exerted protective roles against sepsis-induced myocardial injury.

UTI inhibits NLRP3 and caspase-1 expression in myocardial tissue. NLRP3 inflammasome activation is reflected by the enhanced expression of NLRP3 and caspase-1 (27). Therefore, to verify NLRP3 and caspase-1 inhibition by UTI, mRNA and protein levels were analyzed in all groups. No significant expression differences were observed between control and sham groups $(\mathrm{P}>0.05)$, however the CLP group had higher NLRP3 and caspase-1 expression levels than the other groups $(\mathrm{P}<0.01)$, whereas the high-dose UTI group had lower NLRP3 and caspase- 1 mRNA expression than the low-dose UTI group (P<0.05; Fig. 6A and B). NLRP3 and caspase-1 protein expression levels gradually decreased as the UTI concentration increased $(\mathrm{P}<0.01$; Fig. 7A-C). Thus, UTI significantly inhibited NLRP3 and caspase-1 protein expression.

\section{Discussion}

In the present study, animal models, biochemical assays, histopathological examinations, and mRNA and protein expression analyses were used to elucidate the molecular mechanisms underpinning sepsis-induced myocardial dysfunction, and to investigate the cardioprotective effects of UTI. Firstly, TNF- $\alpha$, IL-1 $\beta$, cTnI and BNP levels were significantly increased and secondly, myocardial tissue pathological alterations were induced, indicating a sepsis-induced myocardial injury model was successfully established.

UTI is widely used to treat acute pancreatitis and pancreatic injury (28-30). In addition to its protease inhibition 
activities, previous studies have also shown UTI exhibits organ-protective effects $(31,32)$, with these effects related to anti-inflammatory and anti-oxidative stress responses. Due to the essential role of anti-inflammatory responses in sepsis-related organ injury pathogenesis, these mechanisms present novel potential strategies for organ dysfunction prognoses and sepsis treatments $(21,33)$. Masuda et al (34) reported that UTI reduced the degree of myocardial injury during sepsis due to its anti-inflammatory and anti-oxidative stress responses.

Inflammatory mediator levels produced during sepsis serve as markers to assess the severity and therapeutic responses to infections $(35,36)$. TNF- $\alpha$ and IL-1 $\beta$ are major inflammatory cytokines produced during sepsis $(37,38)$ and are often used to assess the extent of inflammation (39). TNF- $\alpha$ and IL-1 $\beta$ both have roles in sepsis-induced myocardial injury (40). In the present study, it was observed that myocardial injury was linked to the release of TNF- $\alpha$ and IL-1 $\beta$, whereas UTI decreased these levels, which was similar to results reported in previous studies (41-43).

The cTnI marker is a sensitive indicator of myocardial injury and is mainly released by cardiac myocytes (44). Similarly, BNP is a well-recognized biomarker of myocardial dysfunction and has been used to assess sepsis-induced myocardial dysfunction (45). The findings observed in the current study showed that cTnI and BNP serum levels were significantly elevated in the CLP group, but significantly lower in the UTI groups. These biomarker alterations suggested sepsis exerted adverse effects on the myocardium, whereas UTI provided protection against myocardial injury via anti-inflammatory effects in this rat model.

The animal studies in the present study were performed to verify NLRP3 inflammasome mechanisms during septic myocardial injury; elevated NLRP3 and caspase-1 expression levels in the myocardium were synchronous with TNF- $\alpha$ and IL-1 $\beta$ serum expression levels. Accumulating evidence has indicated that the NLRP3 inflammasome has a role during cardiac dysfunction (46). The NLRP3 inflammasome is composed of NLRP3, ASC and caspase-1, with endogenous cytokines, including TNF- $\alpha$, activating the inflammasome via the $\mathrm{NF}-\kappa \mathrm{B}$ pathway (12). This activation releases IL-1 $\beta$ and promotes inflammatory responses, thereby inducing myocardial injury (41). The present study demonstrated that the NLRP3 inflammasome activation (measured as NLRP3 and caspase-1 activation) was significantly increased in the CLP group compared with control and sham groups, therefore indicating that myocardial injury is related to NLRP3 inflammasome activation.

The effects of UTI on injured myocardium in septic rats was also evaluated in the current study. It was found that various UTI doses reduced NLRP3 and caspase-1 protein expression during myocardial injury induced by CLP, suggesting that UTI suppressed NLRP3 inflammasome activation. Furthermore, when compared with low-dose UTI, the high-dose group displayed an improved outcome as it inhibited the NLRP3 inflammasome to a higher degree. Additionally, a similar trend was observed for IL-1 $\beta$. A previous study also confirmed that NLRP3 inflammasome activation can mediate IL-1 $\beta$ maturation via caspase-1 (47). Taken together, these findings suggested that the protective effects of UTI on the myocardium may be mediated by the downregulation of the NLRP3/caspase-1/IL-1 $\beta$ pathway.

The results of the present study were consistent with previous studies showing that UTI was associated with a reduced risk of mortality in sepsis $(48,49)$. However, high-dose UTI did not show advantages in terms of fatality rates; as the study observation period was relatively short, the humane end points were set at 48 $\mathrm{h}$, therefore if observation times were extended, the results might not have been the same. Additionally, it was observed that clinical severity scores in the low-dose UTI group were significantly higher than the high-dose group; there were statistically significant differences with respect to biomarkers, histopathological examinations, and gene and protein expression levels. Therefore, these findings provided crucial data on the effectiveness of UTI intervention for sepsis-induced myocardial injury treatment.

There are several limitations of the present study. Firstly, the percent survival rate was expressed by Kaplan-Meier survival curve analysis, the sample size of 15 rats per group was relatively small. Secondly, this study mainly focused on the microstructure and molecular biology of sepsis-induced myocardial injury, and echocardiography for the evaluation of septic myocardial injury was not used. Thirdly, two doses of UTI $(5,000 \mathrm{U} / \mathrm{kg}$ as low-dose UTI and $20,000 \mathrm{U} / \mathrm{kg}$ as high-dose UTI) were used to observe the effects on animals, and dose-response curves were not determined to test the effects of UTI on sepsis-induced myocardial injury.

In conclusion, it was demonstrated that UTI potentially exerted beneficial effects by inhibiting inflammatory cytokine release and downregulating NLRP3 inflammasome activation. Similarly, high-dose UTI significantly preserved the myocardium. This study has provided a scientific basis that suggests that UTI may serve as a novel therapeutic strategy targeting the NLRP3/caspase-1/IL-1 $\beta$ signaling pathway during sepsis-induced myocardial injury. Further clinical studies are required to determine its clinical efficacy.

\section{Acknowledgements}

The authors are grateful to Dr Dongmei Hu (School of Statistics, Dalian Medical University, Dalian, China) for her help with the statistical analysis.

\section{Funding}

This work was supported by the Natural Science Foundation of Liaoning Province (grant no. 20180551029), the Scientific Research Project of Liaoning Provincial Department of Education (grant no. LZ2020036) and the National Natural Science Foundation of China (grant no. 81803896).

\section{Availability of data and materials}

All data generated or analyzed during this study are included in this published article.

\section{Authors' contributions}

JQ and YZ designed the study and performed experiments. YZ drafted the manuscript. XX and XG collected and analyzed experimental data. JQ and YZ confirm the authenticity 
of all the raw data. All authors read and approved the final manuscript.

\section{Ethics approval and consent to participate}

This research was approved by the Ethics Committee of Dalian Medical University (approval no. L20160097).

\section{Patient consent for publication}

Not applicable.

\section{Competing interests}

The authors declare that they have no competing interests.

\section{References}

1. Singer M, Deutschman CS, Seymour CW, Shankar-Hari M, Annane D, Bauer M, Bellomo R, Bernard GR, Chiche JD, Coopersmith CM, et al: The third international consensus definitions for sepsis and septic shock (sepsis-3). JAMA 315: 801-810, 2016.

2. Cohen J: The immunopathogenesis of sepsis. Nature 420: 885-891, 2002

3. Turner A, Tsamitros M and Bellomo R: Myocardial cell injury in septic shock. Crit Care Med 27: 1775-1780, 1999.

4. Beesley SJ, Weber G, Sarge T, Nikravan S, Grissom CK, Lanspa MJ, Shahul S and Brown SM: Septic cardiomyopathy. Crit Care Med 46: 625-634, 2018.

5. Blanco J, Muriel-Bombín A, Sagredo V, Taboada F, Gandía F, Tamayo L, Collado J, García-Labattut A, Carriedo D, Valledor M, et al: Incidence, organ dysfunction and mortality in severe sepsis: A Spanish multicentre study. Crit Care 12: R158, 2008.

6. Martinon F, Burns K and Tschopp J: The inflammasome: A molecular platform triggering activation of inflammatory caspases and processing of proIL-beta. Mol Cell 10: 417-426, 2002.

7. Duewell P, Kono H, Rayner KJ and Latz E: NLRP3 inflammasomes are required for atherogenesis and activated by cholesterol crystals. Nature 464: 1357-1361, 2010.

8. Baroja-Mazo A, Martín-Sánchez F, Gomez AI, Martínez CM, Amores-Iniesta1 J, Compan V, Barberà-Cremades $\mathrm{M}$, Yagüe $\mathrm{J}$, Ruiz-Ortiz E, Antón J, et al: The NLRP3 inflammasome is released as a particulate danger signal that amplifies the inflammatory response. Nat Immunol 15: 738-748, 2014.

9. Franklin BS, Bossaller L, De Nardo D, Ratter JM, Stutz A, Engels G, Brenker C, Nordhoff M, Mirandola SR, Al-Amoudi A, et al: The adaptor ASC has extracellular and 'prionoid' activities that propagate inflammation. Nat Immunol 15: 727-737, 2014.

10. Amin J, Boche D and Rakic S: What do we know about the inflammasome in humans? Brain Pathol 27: 192-204, 2017.

11. Sutterwala FS, Haasken S and Cassel SL: Mechanism of NLRP3 inflammasome activation. Ann N Y Acad Sci 1319: 82-95, 2014.

12. Danielski LG, Giustina AD, Bonfante S, Barichello T and Petronilho F: The NLRP3 inflammasome and its role in sepsis development. Inflammation 43: 24-31, 2020.

13. Zhong Y, Lu Y, Yang X, Tang Y, Zhao K, Yuan C and Zhong X: The roles of NLRP3 inflammasome in bacterial infection. Mol Immunol 122: 80-88, 2020.

14. Jiang H, He H, Chen Y, Huang W, Cheng J, Ye J, Wang A, Tao J, Wang C, Liu Q et al: Identification of a selective and direct NLRP3 inhibitor to treat inflammatory disorders. J Exp Med 214: 3219-3238, 2017.

15. Huang Y, Jiang H, Chen Y, Wang X, Yang Y, Tao J, Deng X, Liang $\mathrm{G}$, Zhang $\mathrm{H}$, Jiang $\mathrm{W}$ and Zhou R: Tranilast directly targets NLRP3 to treat inflammasome-driven diseases. EMBO Mol Med 10: e8689, 2018.

16. Zhang P, Tsuchiya K, Kinoshita T, Kushiyama H, Suidasari S, Hatakeyama M, Imura H, Kato N and Takashi S: Vitamin B6 prevents IL-1 $\beta$ protein production by inhibiting NLRP3 inflammasome activation. J Biol Chem 291: 24517-24527, 2016.
17. Li PL: Cardiovascular pathobiology of inflammasomes: Inflammatory machinery and beyond. Antioxid Redox Signal 22: 1079-1083, 2015.

18. Umeadi C, Kandeel F and Al-Abdullah IH: Ulinastatin is a novel protease inhibitor and neutral protease activator. Transplant Proc 40: 387-389, 2008.

19. Xu CE, Zhang MY, Zou CW and Guo L: Evaluation of the pharmacological function of ulinastatin in experimental animals. Molecules 17: 9070-9080, 2012.

20. Xu Q, Yan Q and Chen S: Use of ulinastatin was associated with reduced mortality in critically ill patients with sepsis. J Thorac Dis 11: 1911-1918, 2019.

21. Xu Q, Yan Q and Chen S: Ulinastatin is effective in reducing mortality for critically ill patients with sepsis: A causal mediation analysis. Sci Rep 8: 14360, 2018.

22. National Research Council (US) Committee for the Update of the Guide for the Care and Use of Laboratory Animals: Guide for the Care and Use of Laboratory Animals, 8th edition. National Academies Press (US), Washington, DC, 2011.

23. Rittirsch D, Huber-Lang MS, Flierl MA and Ward PA: Immunodesign of experimental sepsis by cecal ligation and puncture. Nat Protoc 4: 31-36, 2009.

24. Otero-Antón E, González-Quintela A, López-Soto A, López-Ben S, Llovo J and Pérez LF: Cecal ligation and puncture as a model of sepsis in the rat: Influence of the puncture size on mortality, bacteremia, endotoxemia and tumor necrosis factor alpha levels. Eur Surg Res 33: 77-79, 2001.

25. Gonnert FA, Recknagel P, Seidel M, Jbeily N, Dahlke K, Bockmeyer CL, Winning J, Lösche W, Claus RA and Bauer M: Characteristics of clinical sepsis reflected in a reliable and reproducible rodent sepsis model. J Surg Res 170: e123-e134, 2011.

26. Livak KJ and Schmittgen TD: Analysis of relative gene expression data using real-time quantitative PCR and the 2(-Delta Delta C(T)) method. Methods 25: 402-408, 2001.

27. Paik S, Kim JK, Silwal P, Sasakawa C and Jo EK: An update on the regulatory mechanisms of NLRP3 inflammasome activation. Cell Mol Immunol 18: 1141-1160, 2021.

28. Uemura K, Murakami Y, Hayashidani Y, Sueda T, Hashimoto Y, Ohge $\mathrm{H}$ and Sueda T: Randomized clinical trial to assess the efficacy of ulinastatin for postoperative pancreatitis following pancreaticoduodenectomy. J Surg Oncol 98: 309-313, 2008

29. Gao C, Huan J, Li W and Tang J: Protective effects of ulinastatin on pancreatic and renal damage in rats following early scald injury. Burns 35: 547-552, 2009.

30. Feng C, Yang H, Huang S and Li T: Early local drug therapy for pancreatic contusion and laceration. Pancreatology 19: 285-289, 2019.

31. Atal SS and Atal S: Ulinastatin-a newer potential therapeutic option for multiple organ dysfunction syndrome. J Basic Clin Physiol Pharmacol 27: 91-99, 2016.

32. Wang J, Zhou J and Bai S: Combination of glutamine and ulinastatin treatments greatly improves sepsis outcomes. J Inflamm Res 13: 109-115, 2020.

33. Karnad DR, Bhadade R, Verma PK, Moulick ND, Daga MK, Chafekar ND and Iyer S: Intravenous administration of ulinastatin (human urinary trypsin inhibitor) in severe sepsis: A multicenter randomized controlled study. Intensive Care Med 40: 830-838, 2014.

34. Masuda T, Sato K, Noda C, Noda C, Ikeda KM, Matsunaga A, Ogura MN, Shimizu K, Nagasawa H, Matsuyama $N$ and Izumi T: Protective effect of urinary trypsin inhibitor on myocardial mitochondria during hemorrhagic shock and reperfusion. Crit Care Med 31: 1987-1992, 2003.

35. Bozza FA, Salluh JI, Japiassu AM, Soares M, Assis EF, Gomes RN, Bozza MT, Castro-Faria-Neto HC and Bozza PT: Cytokine profiles as markers of disease severity in sepsis: A multiplex analysis. Crit Care 11: R49, 2007.

36. Van der Poll T, Van de Veerdonk FL, Scicluna BP and Netea MG: The immunopathology of sepsis and potential therapeutic targets. Nat Rev Immunol 17: 407-420, 2017.

37. Cannon JG, Tompkins RG, Gelfand JA, Michie HR, Stanford GG, van der Meer JW, Endres S, Lonnemann G, Corsetti J, Chernow B, et al: Circulating interleukin-1 and tumor necrosis factor in septic shock and experimental endotoxin fever. J Infect Dis 161: 79-84, 1990.

38. Girardin E, Grau GE, Dayer JM, Roux-Lombard P and Lambert PH: Tumor necrosis factor and interleukin-1 in the serum of children with severe infectious purpura. N Engl J Med 319: 397-400, 1988. 
39. Hedayat M, Mahmoudi MJ, Rose NR and Rezaei N Proinflammatory cytokines in heart failure: Double-edged swords. Heart Fail Rev 15: 543-562, 2010.

40. Hunter JD and Doddi M: Sepsis and the heart. Br J Anaesth 104: 3-11, 2010.

41. Kumar A, Thota V, Dee L, Olson J, Uretz E and Parrillo JE: Tumor necrosis factor alpha and interleukin lbeta are responsible for in vitro myocardial cell depression induced by human septic shock serum. J Exp Med 183: 949-958, 1996.

42. Cao YZ, Tu YY, Chen X and Liu MH: Protective effect of Ulinastatin against murine models of sepsis: Inhibition of TNF- $\alpha$ and IL- 6 and augmentation of IL-10 and IL-13. Exp Toxicol Pathol 64: 543-547, 2012

43. Hu CL, Li H, Xia JM, Li X, Zeng X, Liao XX, Zhan H, Jing XL and Dai G: Ulinastatin improved cardiac dysfunction after cardiac arrest in New Zealand rabbits. Am J Emerg Med 31: 768-774, 2013.

44. Fromm RE Jr: Cardiac troponins in the intensive care unit: Common causes of increased levels and interpretation. Crit Care Med 35: 584-588, 2007.

45. Charpentier J, Luyt CE, Fulla Y, Vinsonneau C, Cariou A, Grabar S, Dhainaut JF, Mira JP and Chiche JD: Brain natriuretic peptide: A marker of myocardial dysfunction and prognosis during severe sepsis. Crit Care Med 32: 660-665, 2004.
46. Li N, Zhou H, Wu H, Wu Q, Duan M, Deng W and Tang Q: STING-IRF3 contributes to lipopolysaccharide-induced cardiac dysfunction, inflammation, apoptosis and pyroptosis by activating NLRP3. Redox Biol 24: 101215, 2019.

47. He Y, Hara $H$ and Núñez G: Mechanism and regulation of NLRP3 inflammasome activation. Trends Biochem Sci 41: 1012-1021, 2016.

48. Linder A and Russell JA: An exciting candidate therapy for sepsis: Ulinastatin, a urinary protease inhibitor. Intensive Care Med 40: 1164-1167, 2014

49. Wang N, Lui X, Zheng X, Cao H, Wei G, Zhu Y, Fan S, Zhou H and Zheng J: Ulinastatin is a novel candidate drug for sepsis and secondary acute lung injury, evidence from an optimized CLP rat model. Int Immunopharmacol 17: 799-807, 2013.

This work is licensed under a Creative Commons Attribution-NonCommercial-NoDerivatives 4.0 International (CC BY-NC-ND 4.0) License. 